\title{
THE AIST-STRUVE SPACE PROJECT SKY SURVEY
}

\author{
M.S. CHUBEY, I.M. KOPYLOV, D.L. GORSHANOV, \\ I.I. KANAYEV, V.N. YERSHOV, A.E. IL'IN, \\ T.R. KIRIAN AND M.G. VYDREVICH \\ Pulkovo Observatory, Saint-Petersburg, Russia
}

\section{Introduction}

The Struve space astrometric project (Yershov et al. 1995) is being developed by a consortium of Russian space instrumentation institutes in order to extend the Hipparcos satellite reference system (the project initially named $A I S T$, owes its current name to the first director of the Pulkovo Observatory).

The extension of the Hipparcos system means a density of about 100 stars per square degree (at least 4 million stars in the output catalogue). The proper motions of the Hipparcos stars are to be determined with an accuracy of about 0.1 mas year $^{-1}$ due to the epoch difference between two catalogues. The mean accuracy of star positions in the output catalogue is expected to be 0.6 mas which could be achieved by proper design of the satellite (symmetry, smooth rotation, etc.), optics and the micrometer. With a properly designed micrometer (with CCD arrays, special processors for image processing and compressing the data flux to the ground station) it will be possible to observe all objects of the sky down to a definite limiting magnitude. This survey technique assumes no input catalogs to be used for observations.

\section{Micrometer and Photometric System}

One-square degree field of view of the on-board telescopes is planned to be filled with a mosaic of relatively small CCDs ( 28 detectors of $13 \times 15 \mathrm{~mm}$ size each). Some of the CCDs will be covered with color filters (different number of filters for different spectral bands) in order to obtain photometry of stars in the Vilnius 7-band photometric system (Kopylov et al. 1994). This photometric system seems to be optimal for the survey because it provides 
sufficient information for classification of objects and the determination of their physical properties and chemical composition.

Each CCD array with square $16 \times 16 \mu$ pixels will have a rigid adjustable base. The precision of the adjustment is about $\pm 4 \mu$, and it is restricted mainly by the CCD flat surface which does not fit strictly the curvature of the spherical focal surfaces of the Schmidt telescope.

\section{Limiting Magnitude and Accuracy of Observations}

The image is uniformly moving over the field of view because the scanning mode is chosen for the observations. This is one of the most effective ways to survey the sky. The CCDs are planned to be working in the Time Delay and Integration mode, when the accumulated charges are shifted synchronously with the image. The integration time will be approximately 7 seconds for each detector. In total, each transit lasts 35 seconds, and estimations show that the limiting magnitude $V \approx 18^{m}$ is achievable in the Vilnius photometric bands and $\approx 22^{m}$ in the wide band $\lambda \in(300,800 \mathrm{~nm})$. Computer simulations show the accuracy of the CCD observations will be $\leq 0.4$ mas for coordinates of a $V=14^{\mathrm{m}}$ star image, $\leq 3.0$ mas for a $V=18^{m}$ star, $\leq 4 \mathrm{mmag}$ and $\leq 35 \mathrm{mmag}$ in the photometry of 14 and 18-magnitude stars, respectively.

\section{Conclusion}

The described project is intermediate between Hipparcos and the planned GAIA project (Lindegren and Perryman 1995). The Struve output catalogue will be less accurate than that of GAIA, but it could be much easier and faster to do then any interferometric project, and it will give very valuable information on the dynamics and evolution of the stars in our galaxy. The accuracy of the Struve catalog will be higher than that of the Hipparcos. The resulting reference system could be maintained at the one milliarcsecond level for at least $40-50$ years.

\section{References}

I.M.Kopylov, D.L.Gorshanov and M.S.Chubey, 1995. in "Astronomical and Astrophysical Objectives of Sub-Milliarcsecond Optical Astrometry", 327-330. E.Høg \& P.K.Seidelmann (eds.), Kluwer Publ.

L.Lindegren, M.A.C.Perryman, 1995. in "Future Possibilities for Astrometry in Space", 23-34, ESA SP-379.

V.N.Yershov, M.S.Chubey, A.E.Ill'in, I.M.Kopylov, D.L.Gorshanov, I.I.Kanayev, and T.R.Kirian, 1995, "Project STRUVE", "Glagol," St-Petersburg, 272 p., (in Russian). 